\title{
Demo: WiART - Visualize and Interact with Wireless Networks using Augmented Reality
}

\author{
Danh H. Nguyen, James Chacko, Logan Henderson, Anton Paatelma*, \\ Harri Saarnisaari* Nagarajan Kandasamy, and Kapil R. Dandekar \\ Drexel University, Philadelphia PA, USA \\ *Center for Wireless Communications, University of Oulu, Finland \\ \{dnguyen, jjc652, ljh66, kandasamy, dandekar\}@drexel.edu \\ \{anton.paatelma, harri.saarnisaari\}@ee.oulu.fi
}

\begin{abstract}
With the increasing programmability and fast-paced dynamics of modern wireless systems, it has become more difficult to gain timely insights into wireless network operations. In this demonstration ${ }^{1}$ we present WiART, an augmented reality framework to help visualize and interact with wireless network activities in real time. WiART collects real-time radio and network statistics from participating network devices and depicts them on users' mobile devices in an intuitive way, leveraging the virtual information overlay of augmented reality. Specifically in our current implementation, WiART takes inputs from a cognitive radio link controlling beam-steerable reconfigurable antennas and annotates on a live mobile screen the active pre-measured radiation patterns. In the reverse flow, WiART enables users to select desired antenna radiation patterns directly in the mobile app and observe their effects on link performance in real time. These capabilities add an unprecedented level of instant visualization and interaction with wireless activities and provides valuable insights into the dynamics of a reconfigurable antenna-based cognitive radio network.
\end{abstract}

\section{CCS Concepts}

- Networks $\rightarrow$ Network monitoring; • Human-centered computing $\rightarrow$ Mixed / augmented reality; Visualization toolkits;

\section{INTRODUCTION}

With the advent of software-defined radios, software-defined networks, and cloud RANs, wireless networking systems have become increasingly programmable. However, this programmability also makes it difficult to gain timely insights into wireless network operations. For example, in dense smallcell deployments, a mobile user can be handed over among a group of base stations multiple times in a short window (e.g. seconds), and multiple base stations can cooperate with each other to jointly serve the user, using mechanisms such

\footnotetext{
${ }^{1}$ Demo video available at https://youtu.be/saOKWRpYyXo

Permission to make digital or hard copies of part or all of this work for personal or classroom use is granted without fee provided that copies are not made or distributed for profit or commercial advantage and that copies bear this notice and the full citation on the first page. Copyrights for third-party components of this work must be honored. For all other uses, contact the owner/author(s).

MobiCom'16 October 03-07, 2016, New York City, NY, USA

(C) 2016 Copyright held by the owner/author(s).

ACM ISBN 978-1-4503-4226-1/16/10.

DOI: http://dx.doi.org/10.1145/2973750.2985626
}

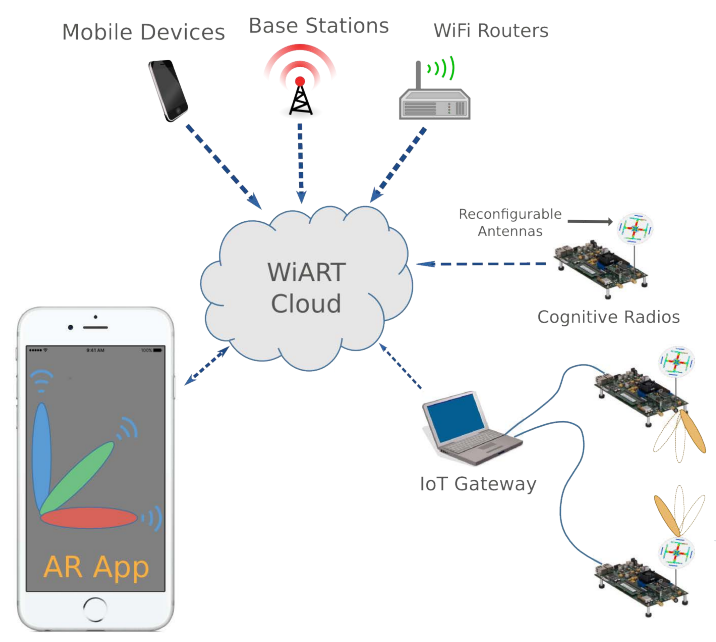

Figure 1: WiART system architecture

as coordinated multipoint (CoMP) and beamforming. This fast-paced dynamic, coupled with the fact that RF electromagnetic signals are invisible and therefore hard to visualize, hinders the ability to conduct wireless research and education with user in the loop, providing inputs and observing real-time system responses. Without a physically intuitive way to visualize and interact with networks and signals propagating through the medium, wireless transmissions often go unaccounted for, and we resort to mathematical analyses or after-the-fact examinations to gain a better understanding of wireless network dynamics.

In this demo, we present the Wireless Augmented Reality Toolkit (WiART), a mobile augmented reality framework for visualization and interaction with wireless networks. WiART extends our previous effort on a visualization method for reconfigurable antennas [2] into a two-way interaction suite that enables users to both visualize and control wireless transmissions from mobile devices. WiART boasts a novel user interface that leverages augmented reality (AR) to blend information from the real and virtual realms, allowing for deeper understanding and controls of wireless activities without resorting to the traditional terminal and web frontends. Though receiving widespread attention lately, AR has not found many applications in the context of wireless connectivity, mainly due to the difficulty of sensing and observing highspeed wireless signals in real time. WiART addresses these challenges using high-performance software radios, while remaining extensible to aid in the development and evaluation of future wireless technologies. 


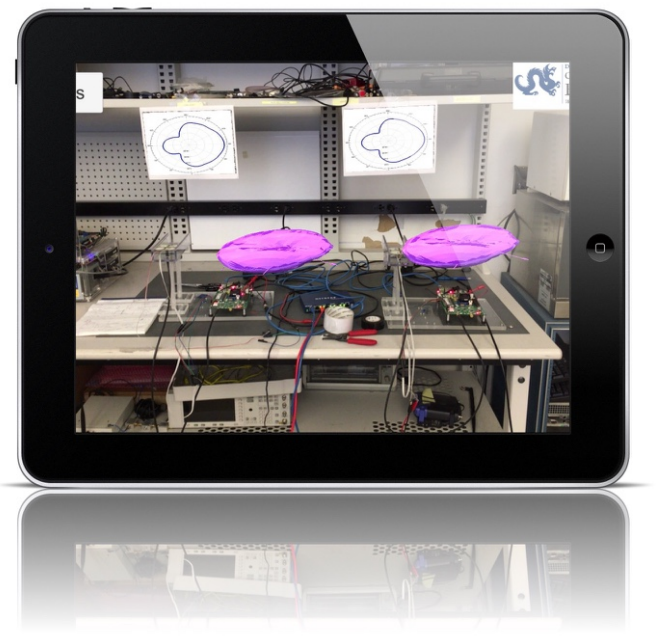

Figure 2: WiART mobile frontend. The purple antenna beams are annotated by the AR toolkit and correspond to pre-measured radiation patterns.

WiART's real-time interactivity is a combination of mobile computer vision, interactive game play, and a web framework. WiART collects real-time radio and network statistics from all participating network devices (mobiles, base stations, routers, etc.) and depicts them on users' mobile devices in an intuitive way, leveraging digital imagery overlay of the real world. The underlay software radio nodes are also necessary to control the reconfigurable antennas and supply useful real-time operating information. An overview of the complete WiART framework is depicted in Fig. 1.

\section{SYSTEM IMPLEMENTATION}

WiART Mobile Frontend: WiART's frontend builds upon the Vuforia mobile AR toolkit and Unity game engine. The AR toolkit allows for for WiART's integration with the real world, while Unity controls the dynamics of virtual objects, including 3D models of the antenna patterns. Reconfigurable antennas are detected spatially through physical image markers placed directly above them. These markers correspond to game objects with well defined positions in the Unity game scene. We once again leverage Vuforia APIs to perform object recognition. Once the reconfigurable antennas are detected by Unity, we add pre-measured 2D and $3 \mathrm{D}$ radiation pattern plots at predefined offsets to the image markers and dynamically control them through game action scripts. The $2 \mathrm{D}$ antenna patterns, measured previously in an anechoic chamber, are overlaid on top of the image markers. The 3D antenna pattern objects were extracted from HFSS simulation and imported into Unity as game objects. At this point the mobile app can connect to WiART's cloud backend and update antenna radiation patterns based on real-time inputs from the underlay cognitive radio link. A snapshot of the mobile frontend is shown in Fig. 2.

WiART Network/Radio Backend: WiART's backend consists of the actual network devices, such as the cognitive radio nodes used in this demo, as well as a cloud-based platform to collect and serve real-time antenna pattern information and other network metrics. The backend cloud interacts directly with Internet-enabled network devices via REST APIs. For non Internet-enabled devices, such as wire-

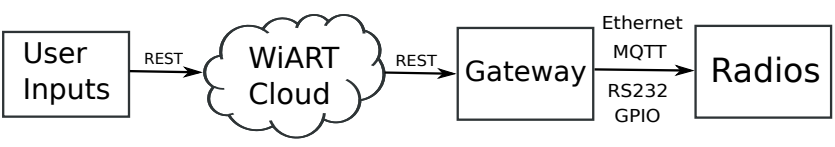

Figure 3: WiART remote radio configuration update flow

less sensors and radios with limited processing capabilities, an Internet-of-Things (IoT) gateway can be used as a middle man. The gateway uses more lightweight messaging protocols, such as Ethernet, MQTT, RS232, or GPIO, to relay messages between the radio devices and WiART cloud. An example WiART remote radio configuration flow is depicted in Fig. 3.

Our demo system employs the planar reconfigurable Alfordloop antennas with integrated control circuitry [3], designed by Drexel Wireless System Laboratory. To control the antennas, we develop a cognitive network link on the Wireless open-Access Research Platform (WARP) [1]. Our implementation leverages WARP's real-time 802.11 physical layer and a custom TDMA-style MAC with machine learning logic for selecting favorable antenna states to boost link performance. An IoT gateway, implemented in Python, communicates with the radio nodes via serial COM ports to collect antenna and throughput statistics, as well as propagates user controls from the mobile app. WiART's frontend polls the backend cloud via REST requests to capture and update the network-wide antenna configurations.

\section{DEMONSTRATION}

We demonstrate WiART using two WARP cognitive radio nodes, both equipped with reconfigurable antennas, communicating in $\mathrm{WiFi}$ channel $14(2.484 \mathrm{GHz})$. A third radio will be set up to turn on and off at times to generate upand downlink interference in the channel, thereby forcing the cognitive radios to adapt their antenna patterns to improve link throughputs. Conference attendees can download and install the WiART mobile app, which will be made available on both iOS and Android app stores, to observe the dynamic antenna patterns and control them on the fly. Link throughputs will be plotted on a monitor screen and in the mobile app at all times to observe the effects of different antenna radiation patterns.

We are self-sufficient in our equipment need for the demo. However, we will need a large (preferrably rollable) monitor, a VGA cable, a 6-foot table, two poster stands, power outlet, and both wired (for the gateway) and wireless (for mobile devices) Internet access. The total setup time is 1 hour.

\section{Acknowledgments}

This work was supported by the National Science Foundation under Grants No. 1457306 and 1241631.

\section{REFERENCES}

[1] WARP Project. http://warpproject.org.

[2] D. H. Nguyen, L. Henderson, J. Chacko, C. Sahin, A. Paatelma, H. Saarnisaari, N. Kandasamy, and K. R. Dandekar. BeamViewer : Visualization of Dynamic Antenna Radiation Patterns using Augmented Reality. In Proc. of IEEE INFOCOM WKSHPS' 16, 2016.

[3] D. Patron and K. R. Dandekar. Planar reconfigurable antenna with integrated switching control circuitry. In Proc. of the 8th European Conference on Antennas and Propagation (EuCAP 2014), pages 2737-2740, 2014. 